\title{
Joint Signal Detection and Classification Based on First-Order Cyclostationarity For Cognitive Radios
}

\author{
O. A. Dobre, ${ }^{1}$ S. Rajan, ${ }^{2}$ and R. Inkol ${ }^{2}$ \\ ${ }^{1}$ Faculty of Engineering and Applied Science, Memorial University of Newfoundland, \\ 300 Prince Philip Dr., St. John's, NL, Canada A1B $3 X 5$ \\ ${ }^{2}$ Defence Research and Development Canada, 3701 Carling Avenue, Ottawa, ON, Canada K1A 0Z4
}

Correspondence should be addressed to O. A. Dobre, odobre@mun.ca

Received 15 February 2009; Revised 1 June 2009; Accepted 8 July 2009

Recommended by R. Chandramouli

\begin{abstract}
The sensing of the radio frequency environment has important commercial and military applications and is fundamental to the concept of cognitive radio. The detection and classification of low signal-to-noise ratio signals with relaxed a priori information on their parameters are essential prerequisites to the demodulation of an intercepted signal. This paper proposes an algorithm based on first-order cyclostationarity for the joint detection and classification of frequency shift keying (FSK) and amplitudemodulated (AM) signals. A theoretical analysis of the algorithm performance is also presented and the results compared against a performance benchmark based on the use of limited assumed a priori information on signal parameters at the receive-side. The proposed algorithm has the advantage that it avoids the need for carrier and timing recovery and the estimation of signal and noise powers.
\end{abstract}

Copyright (C) 2009 O. A. Dobre et al. This is an open access article distributed under the Creative Commons Attribution License, which permits unrestricted use, distribution, and reproduction in any medium, provided the original work is properly cited.

\section{Introduction}

A cognitive radio is an intelligent wireless communication system capable of sensing and adapting to its radio frequency environment. The core idea, first introduced by Joseph Mitola III in his doctoral dissertation [1], is to opportunistically search for and exploit unoccupied portions of the spectrum $[1,2]$. Since much of the spectrum allocated to licensed services is sparsely occupied at any given time [3], such a strategy has the potential to meet the growing demands for spectrum access, efficiency, and reliability in commercial wireless systems. Intelligent radios also have military applications, such as the opportunistic intercept of signals by electronic warfare systems [4]. A major issue in such radios is the detection and classification of low signal-to-noise ratio (SNR) signals with relaxed a priori information on their parameters.

Because of their ease of implementation and the large amount of legacy communications equipment in use, amplitude and frequency shift keying modulation (AM and FSK) techniques continue to be widely employed, particularly in the VHF and UHF bands. Consequently, considerable work has been carried out on techniques for the classification of
FSK and AM signals. Likelihood-based (LB) and featurebased (FB) approaches were the subject of extensive studies in [4-11]. The first approach is based on the likelihood function of the received signal with a likelihood ratio test being used for the classification decision, whereas the second approach is based on the idea that a specific modulation type can be identified by testing for the presence of a suitably chosen set of features extracted from the received signal. Both approaches have been investigated for FSK signal recognition. The LB approach, studied for $M$-ary FSK signals by Beidas and Weber in $[5,6]$, requires signal parameter information, such as symbol rate and frequency deviation. The same authors also presented a theoretical framework linking higher-order correlation domain with the LB approach and used this to construct a time domain correlation-based classification algorithm based on the likelihood function $[5,6]$. In comparison with the LB approach, the correlation-based algorithm is relatively insensitive to carrier frequency offsets. However, the complexity and computational cost of the algorithm are increased when a priori knowledge of the symbol timing is not available. The algorithms proposed by Hsue and Soliman [7] and Ho et al. [8] required timing recovery and 
estimation of the SNR. The algorithm proposed by Hsue and Soliman was based on the histogram of the zero-crossing interval, while that of Ho and others used the magnitude of wavelet transform. Rosti and Koivunen [9] proposed an algorithm based on the mean of the complex signal envelope; their approach also assumed a priori knowledge of the symbol timing. The $M$-ary FSK signal classification algorithm proposed by $\mathrm{Yu}$ et al. [10] was based on the Fourier transform of the signal. Given reasonable a priori information about the signal, this algorithm was reported to have performed well for positive SNRs. Another scheme for FSK and AM signal classification, described in [11], used the statistics of the instantaneous amplitude and frequency with the decision-making reliant on SNR dependent thresholds. However, these approaches involve various limitations and complications, particularly with respect to requirements for the measurement or a priori knowledge of signal parameters.

For more than two decades, the cyclostationary properties of signals have been explored for signal intercept [12], modulation classification [13-18], parameter estimation [19], source separation [20], and other applications. Recently, second-order cyclostationarity was investigated in the context of spectrum sensing and awareness for cognitive radio [21-26]. In contrast with these earlier investigations, this paper focuses on the application of first-order signal cyclostationarity to the joint detection and classification of band-limited FSK and AM signals affected by additive Gaussian noise and phase, frequency, and time delay offsets. A first-order cyclic moment-based algorithm is proposed, which requires only approximate information about the signal bandwidth and carrier frequency. Unlike previously reported approaches, the proposed algorithm does not require the measurement or a priori knowledge of signal parameters such as signal and noise power, carrier phase and frequency offset, and symbol timing. A benchmark is also developed to provide a standard for assessing the performance of the proposed classifier. The connection between this algorithm and the $M$-ary FSK classification algorithm in [10] is also shown and a brief discussion of its computational complexity provided. The rest of the paper is organized as follows. The models of the signals of interest and their first-order cyclostationarity are presented in Sections 2 and 3, respectively. The proposed algorithm and benchmark are introduced in Section 4, while a theoretical analysis of their performance is discussed in Section 5. Numerical results are shown in Section 6. Finally, conclusions are drawn in Section 7. Derivations related to the first-order signal cyclostationarity and the presentation of a cyclostationarity test used with the proposed algorithm are provided in Appendices $\mathrm{A}$ and $\mathrm{B}$, respectively. Note that the results in this paper have been partially presented by the authors in $[27,28]$.

\section{Signal Models}

Using approximate information about the signal bandwidth and carrier frequency, the received signal is down-converted to baseband, and the out-of-band noise is removed by an appropriate filter to yield

$$
r(t)=s(t)+w(t)
$$

where $w(t)$ represents additive zero mean Gaussian noise, and $s(t)$ represents a signal having one of the following possible modulations: (i) FSK modulation, (ii) AM modulation, (iii) Single side-band (SSB) amplitude modulation, (iv) Double side-band (DSB) amplitude modulation, (v) Single-carrier linear digital (SCLD) modulation (such as $M$-ary phase shift keying (PSK) or quadrature amplitude modulation (QAM)), (vi) Cyclically prefixed SCLD (CPSCLD) modulation. Although not explicitly shown, $s(t)$ is also affected by phase, frequency, and time delay offsets. For an FSK signal, $s(t)$ is expressed as

$$
s(t)=\mathcal{A} e^{j \theta} e^{j 2 \pi \Delta f t} \sum_{i} e^{j 2 \pi f_{\Delta} s_{i}\left(t-i T-t_{0}\right)} g\left(t-i T-t_{0}\right),
$$

where $\mathcal{A}$ is the amplitude, $\theta$ and $\Delta f$ represent the phase and frequency offsets, respectively, $f_{\Delta}$ is the frequency deviation, $T$ is the symbol period (for simplicity of notation, $T$ denotes the symbol period of $M$-FSK signals, regardless the modulation order, $M)$, $t_{0}$ is the time delay, $g(t)=u_{T}(t) \otimes g^{(\mathrm{rec})}(t)$ is the signal pulse shape, with $u_{T}(t)$ representing a rectangular pulse of unit amplitude and duration $T, \otimes$ the convolution operator, and $g^{(\mathrm{rec})}(t)$ the impulse response of the equivalent lowpass receive filter, $s_{i}$ is the symbol transmitted within the $i$ th period, and $j=\sqrt{-1}$. The data symbols, $\left\{s_{i}\right\}$, are assumed to be zero-mean independent and identically distributed random variables, with values drawn from the alphabet corresponding to the $M$-FSK modulation, that is, $s_{i} \in A_{M-\mathrm{FSK}}=\left\{\widetilde{s}_{m}: \widetilde{s}_{m}=2 m-1-M, m=1, \ldots, M\right\}$, with a power of 2 modulation order, $M$.

For an AM signal [29], $s(t)$ can be expressed as

$$
s(t)=\mathcal{A} e^{j \theta} e^{j 2 \pi \Delta f t}\left(g^{(\mathrm{rec})}(0)+\mu_{A} x\left(t-t_{0}\right)\right),
$$

where $g^{(\mathrm{rec})}(0)$ is the Fourier transform of $g^{(\mathrm{rec})}(t)$ at zero frequency, with $g^{(\mathrm{rec})}(0)=1, \mu_{A}$ is the modulation index, $x(t)=m(t) \otimes g^{(\mathrm{rec})}(t)$, and $m(t)$ is the zero-mean realvalued band-limited modulating signal.

The signal $r(t)$ is sampled at a sampling rate $f_{s}$ and normalized with respect to the power of the noisy signal at the output of receive filter, yielding the discrete-time normalized signal

$$
r[k]=\frac{\left.r(t)\right|_{t=k f_{s}^{-1}}}{\sqrt{8+\mathcal{N}}}
$$

where $\delta$ and $\mathcal{N}$ are the signal and noise powers at the output of receive filter, respectively. Note that an estimate of the noisy signal power can be straightforwardly obtained from the sample sequence and does not require separate estimation of the signal and noise powers. The receive filter allows the significant spectral components of the signal to pass through unattenuated, and, as a result, the signal power at the output of the filter is approximately equal to the input power. Consequently, the signal amplitude, $\mathcal{A}$, 
can be approximately expressed as $\sqrt{8}$ for the FSK signals, and as $\sqrt{8 /\left(1+\mu_{A}^{2} \mathrm{E}\left[m^{2}(t)\right]\right)}$ for the AM signals, where $\mathrm{E}[\cdot]$ is the statistical expectation. In addition, the modulation constraint $\left|\mu_{A} m(t)\right| \leq 1$ for AM signals [29] results in $\left|\mu_{A}^{2} \mathrm{E}\left[m^{2}(t)\right]\right| \leq 1$, which yields amplitude values between $\sqrt{8 / 2}$ and $\sqrt{8}$.

Models of SSB, DSB, SCLD, and CP-SCLD band-limited signals affected by phase, frequency, and time delay offsets are given in various publications, for example, [13-18].

\section{First-Order Signal Cyclostationarity}

3.1. Fundamental Concepts. Let $r(t)$ be a first-order cyclostationary process. The first-order time-varying moment of $r(t)$, defined as $\tilde{m}_{r}(t)=\mathrm{E}[r(t)]$, is an (almost) periodic function of time and accepts a Fourier series expansion as [30]

$$
\tilde{m}_{r}(t)=\sum_{\tilde{\alpha} \in \tilde{\kappa}} \tilde{m}_{r}(\tilde{\alpha}) e^{j 2 \pi \tilde{\alpha} t}
$$

where $\tilde{\kappa}=\left\{\tilde{\alpha}: \tilde{\mathrm{m}}_{r}(\tilde{\alpha}) \neq 0\right\}$ represents the set of first-order cycle frequencies (CFs), and $\tilde{\mathrm{m}}_{r}(\tilde{\alpha})$ is the first-order cyclic moment (CM) at CF $\tilde{\alpha}$, defined as

$$
\tilde{m}_{r}(\tilde{\alpha})=\lim _{I \rightarrow \infty} I^{-1} \int_{-I / 2}^{I / 2} \tilde{m}_{r}(t) e^{-j 2 \pi \tilde{\alpha} t} d t .
$$

For the discrete-time signal $r[k]=\left.r(t)\right|_{t=k f_{s}^{-1}}$, obtained by sampling the continuous-time signal $r(t)$ at a sampling rate $f_{s}$, the first-order CM and corresponding set of CFs are, respectively, given as (under the assumption of no aliasing) [31]

$$
\begin{gathered}
m_{r}(\alpha)=\tilde{m}_{r}\left(\alpha f_{s}\right), \\
\kappa=\left\{\alpha: \alpha \in\left[-\frac{1}{2}, \frac{1}{2}\right), \alpha=\tilde{\alpha} f_{s}^{-1}, m_{r}(\alpha) \neq 0\right\} .
\end{gathered}
$$

The estimator of the first-order CM at CF $\alpha$, based on $K$ samples, is given as [32]

$$
\hat{m}_{r}^{(K)}(\alpha)=K^{-1} \sum_{k=0}^{K-1} r[k] e^{-j 2 \pi \alpha k} .
$$

3.2. First-Order Cyclostationarity of the Signals of Interest. According to the results derived in Appendix A, if $f_{\Delta}=l T^{-1}$, with $l$ as an integer, then the received FSK signal exhibits firstorder cyclostationarity. The first-order CM of the discretetime normalized signal at CF $\alpha$, and the set of first-order CFs are given, respectively, as

$$
\begin{gathered}
m_{r}(\alpha)=\frac{e^{j \theta} e^{-j 2 \pi \gamma f_{s} t_{0}} \mathcal{A}}{M \sqrt{8+\mathcal{N}}}, \\
\kappa=\left\{\alpha \in\left[-\frac{1}{2}, \frac{1}{2}\right): \alpha=\gamma+\Delta f f_{s}^{-1},\right. \\
\text { with } \gamma=p T^{-1} f_{s}^{-1}, \\
p= \pm l, \ldots, \pm(\mathrm{M}-1) l\} .
\end{gathered}
$$

Note that the first-order CM at frequencies other than CFs equals zero. On the other hand, the first-order CM at CF $\alpha, m_{r}(\alpha)$, depends on the modulation order $M$, phase, $\theta$, time delay, $t_{0}$, frequency deviation, $f_{\Delta}$ (through $\gamma$ ), alphabet, $A_{M-\text { FSK }}$ (through $\gamma$ ), and SNR (with $\mathcal{A}$ approximately equal to $\sqrt{8}$ and SNR defined as $8 / \mathcal{N})$. Based on $(10)$, it is straightforward that the magnitude of the first-order CM at $\mathrm{CF} \alpha$ is given as

$$
\left|m_{r}(\alpha)\right|=\frac{\mathcal{A}}{M \sqrt{8+\mathcal{N}}}
$$

and depends only on the modulation order $M$ and SNR (with $\mathcal{A}$ approximately equal to $\sqrt{8}$ ). It is noteworthy that the CM magnitude decreases with an increase in $M$ and a decrease in the SNR. In addition, according to (11), the number of first-order CFs is equal to the modulation order, $M$, and for any given $M$, the CFs depend on the frequency offset, $\Delta f$, frequency deviation, $f_{\Delta}$ (through $\gamma$ ), alphabet, $A_{M-\mathrm{FSK}}$ (through $\gamma$ ), and sampling frequency, $f_{s}$. Also from (11), it can be easily seen that the distance between any two adjacent CFs equals $2 f_{\Delta} f_{s}^{-1}$.

The first-order CM of the discrete-time normalized AM signals at CF $\alpha$ and the set of first-order CFs are given respectively, as (see Appendix A)

$$
\begin{aligned}
m_{r}(\alpha) & =\frac{\mathcal{A} e^{j \theta}}{\sqrt{(\mathcal{S}+\mathcal{N})}}, \\
\kappa & =\left\{\alpha \in\left[-\frac{1}{2}, \frac{1}{2}\right): \alpha=\Delta f f_{s}^{-1}\right\} .
\end{aligned}
$$

As for the FSK signals, the first-order CM of the AM signals at frequencies other than CFs is equal to zero. Based on (13), the magnitude of the first-order CM at CF $\alpha$ becomes

$$
\left|m_{r}(\alpha)\right|=\frac{\mathcal{A}}{\sqrt{\mathscr{S}+\mathcal{N}}} .
$$

With the signal amplitude, $\mathcal{A}$, approximately given by $\sqrt{8 /\left(1+\mu_{A}^{2} \mathrm{E}\left[m^{2}(\mathrm{t})\right]\right)}$, one can notice that the magnitude of the first-order CM at CF $\alpha$ depends on the SNR (it decreases with a decrease in the SNR), modulation index, $\mu_{A}$, and power of the modulating signal, $\mathrm{E}\left[\mathrm{m}^{2}(t)\right]$ and takes values between $\sqrt{\delta / 2(\delta+\mathcal{N})}$ and $\sqrt{\delta /(\delta+\mathcal{N})}$. In addition, based on (14), there is a single first-order CF, which depends on the carrier frequency offset, $\Delta f$, and sampling frequency, $f_{s}$.

According to results presented in [13-18], one can infer that SSB, DSB, SCLD, and CP-SCLD signals do not exhibit first-order cyclostationarity. For example, by using the closed-form expressions for the temporal parameters of the $n$ th-order cyclostationarity of SCLD signals [16], it is straightforward to obtain the first-order CM at CF $\alpha$ as

$$
m_{r}(\alpha)=\mathcal{A} m_{s} \rho^{-1} e^{j \theta} e^{-j 2 \pi \alpha \rho t_{0} T^{-1}} \sum_{k} g[k] e^{-j 2 \pi \alpha k},
$$

with $m_{s}$ as the first-order moment of the signal constellation points and $\rho$ as the oversampling factor. For symmetric signal constellations, such as PSK and QAM, $m_{s}$ equals zero, which in turn leads to the nullity of $m_{r}(\alpha)$ for any $\alpha$. In addition to SSB, DSB, SCLD, and CP-SCLD signals, the noise, $w(t)$, being a zero-mean stationary process, does not exhibit firstorder cyclostationarity. 


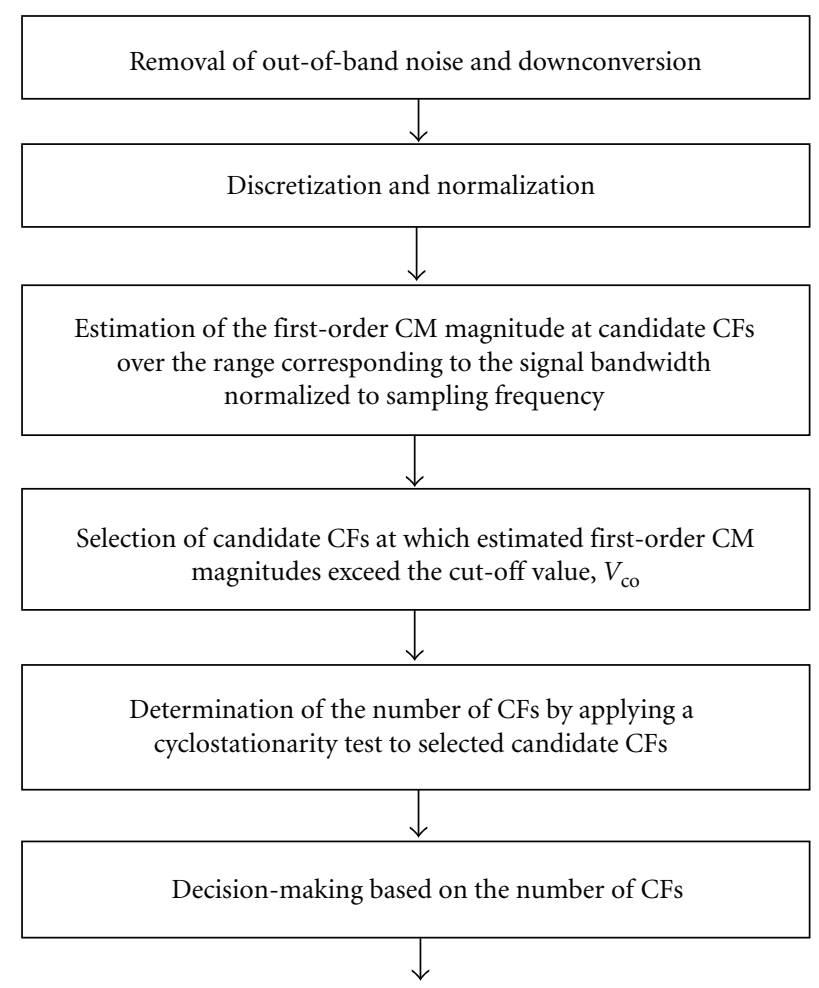

FSK signal of modulation order, $M$, AM signal, or noise or other signals, such as SSB, DSB, SCLD, and CP-SCLD

Figure 1: Block diagram of the proposed first-order cyclostationarity-based joint detection and classification algorithm.

\section{First-Order Cyclostationarity-Based Joint Detection and Classification of FSK and AM Signals}

The existence and number of first-order CFs are, respectively, exploited for the detection and classification of FSK and AM signals. A first-order cyclostationarity-based joint signal detection and classification algorithm is proposed, with relaxed a priori information on the parameters of the received signal. Furthermore, a benchmark is developed under the assumption that a priori information on the signal parameters is available at the receive-side.

4.1. Proposed Algorithm. With the proposed algorithm, the joint detection and classification of FSK and AM signals is formulated as a multiple-hypothesis testing problem, as follows: (i) the received signal is AM if a single first-order CF is detected; (ii) the received signal is 2-FSK if two firstorder CFs are detected; (iii) the received signal is $M$-FSK $\left(M=2^{m}, M \geq 4\right)$ if the number of first-order CFs which are detected belongs to the interval $\left[2^{m-1}+1,2^{m}\right]$ (A simple majority decision criterion is applied here. More complicated criteria, which can take into account the distance between CFs can be conceived. Obviously, diverse criteria can lead to different performance. This will be studied in future work.); (iv) there is no signal (only noise) or the signal is SSB, DSB,
SCLD, or CP-SCLD, if no first-order CFs are detected. To further detect and classify the latter signals, second- and higher-order signal cyclostationarity can be exploited [1318 .

The proposed algorithm consists of the following two steps.

Step 1. The magnitude of the first-order CM of the normalized signal is estimated at candidate CFs, $\alpha^{\prime}$, over a range corresponding to the bandwidth normalized to the sampling rate, and based on a $K$ sample observation interval. According to the theoretical derivations, the first-order CM magnitude for FSK and AM signals is nonzero only at CFs given in (11) and (14), respectively, whereas for noise and another signals this is zero at all CF candidates. Note that these results are obtained for an infinite observation interval. When estimation is performed based on a finite length data sequence ( $K$ samples), nonzero values are attained for the first-order CM magnitude of both FSK and AM at candidates other than CFs, while such values are achieved at all CF candidates for noise and the other signals. Thus, estimates obtained using finite length data sequences result in the presence of a noise floor. Nevertheless, these nonzero values are statistically insignificant. On the other hand, for both FSK and AM signals, the first-order CM magnitude at CFs decreases with a decrease in SNR, and thus, below a certain SNR, this becomes comparable with the nonzero statistically insignificant values. A cutoff value, $V_{\mathrm{co}}$, is set, and candidate CFs which correspond to a CM magnitude above or equal to $V_{\text {co }}$ are selected for testing in the next step. (Extensive simulations were run to investigate the values of the noise floor for diverse observation intervals, SNRs, sampling frequencies, and signal parameters. Based on these results, cutoff values were set such that most of the statistically insignificant peaks lie below these values (see Section 6 for details). A rigorous mathematical analysis of the noise floor distribution, to be employed for the cutoff value setting, is beyond the scope of this paper and will be addressed in future work.) By using (12) and (15), one can obtain the theoretical value of the SNR for which the first-order CM magnitude at CFs equals $V_{\text {co }}$ for the $M$-FSK and AM signals, respectively. The notation $\mathrm{SNR}_{\mathrm{co}}$ will be subsequently used for this $\mathrm{SNR}$. It is noteworthy that for SNRs well above $\mathrm{SNR}_{\text {co }}$, peaks corresponding to all CFs will be tested, whereas for SNRs well below $\mathrm{SNR}_{\mathrm{co}}$, these will lie below the cutoff value, $\mathrm{V}_{\mathrm{co}}$, and be missed. Examples are given in Section 6.

Step 2. The cyclostationarity test developed in [33] is used to check whether or not the candidate CFs selected in Step 1 of the algorithm are indeed CFs. A detailed description of this test is provided in Appendix B. A first-order CM-based statistic is estimated at each candidate CF and compared against a threshold. The threshold is set from a given (asymptotic) probability of false alarm. This is defined as the probability to decide that a candidate CF is a CF when it is actually not, under the assumption that the observation 


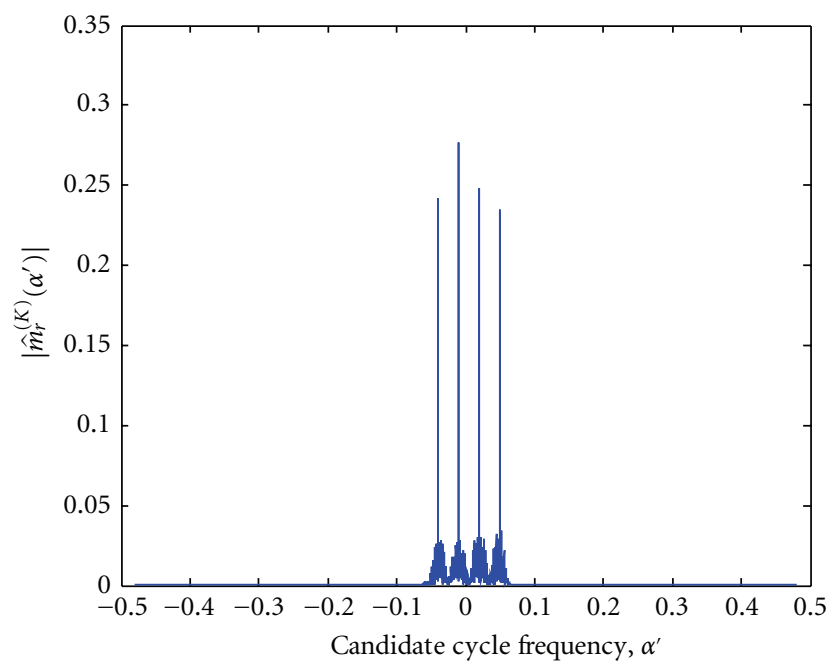

(a)

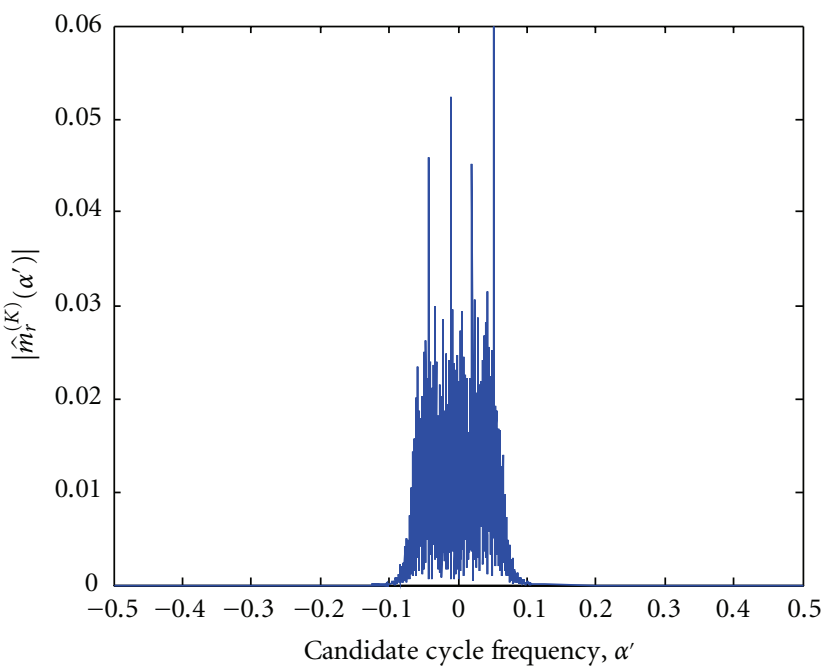

(c)

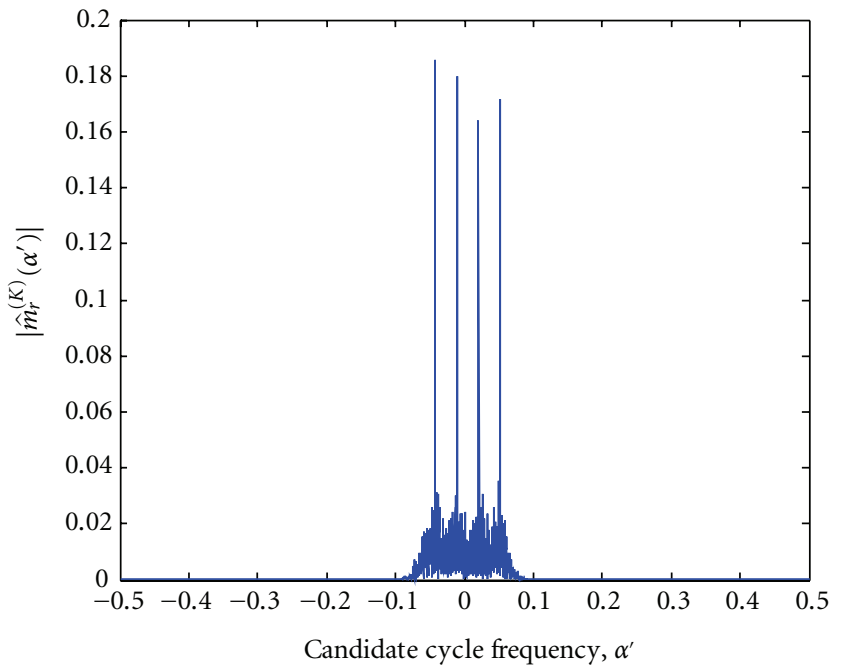

(b)

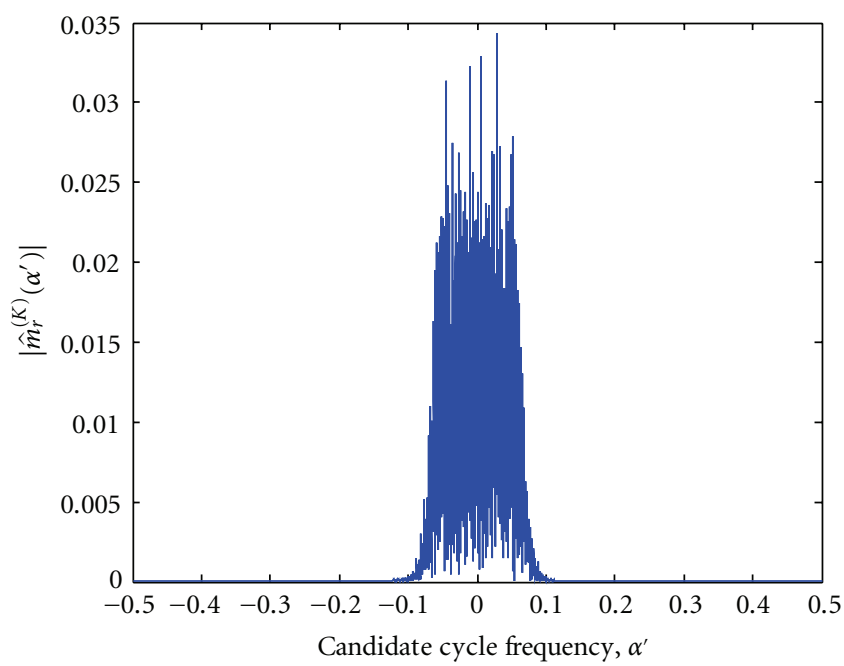

(d)

Figure 2: The magnitudes of the first-order estimated CM of the 4-FSK signal, at candidate CFs $\alpha^{\prime}, \alpha^{\prime} \in[-1 / 2,1 / 2)$, for (a) $20 \mathrm{~dB}$ SNR, (b) $0 \mathrm{~dB}$ SNR, (c) $-13 \mathrm{~dB}$ SNR, and (d) $-20 \mathrm{~dB}$ SNR.

interval goes to infinity, and is calculated based on the (asymptotic) chi-squared distribution of the test statistic at non-CFs. If the estimated statistic at a candidate CF exceeds the threshold, then the candidate is decided to be a CF. The number of CFs is finally employed to make a decision on the signal detection and classification.

The block diagram of the proposed algorithm is shown in Figure 1. Note that the algorithm requires only minimal a priori information on the parameters of the signal.

4.2. Proposed Benchmark. The proposed benchmark is developed under the assumption that the first-order CFs of the signals of interest are known at the receive-side. These CFs are tested with the aforementioned cyclostationarity test and the same decision criterion is applied. As such, the joint detection and classification of FSK and AM signals is formulated as a multiple hypothesis-testing problem, as follows: (i) the received signal is AM if corresponding (known) first-order CF is detected; (ii) the received signal is 2-FSK if the two corresponding (known) first-order CFs are detected; (iii) the received signal is $M$-FSK $\left(M=2^{m}, M \geq 4\right)$ if at least $2^{m-1}+1$ out of the $M$ corresponding (known) firstorder CFs are detected; (iv) there is no signal (only noise) or the signal is SSB, DSB, SCLD, CP-SCLD, if no first-order CF is detected.

\section{Theoretical Performance Analysis}

The proposed algorithm involves the steps of finding candidate CFs at which the magnitudes of estimated first-order 


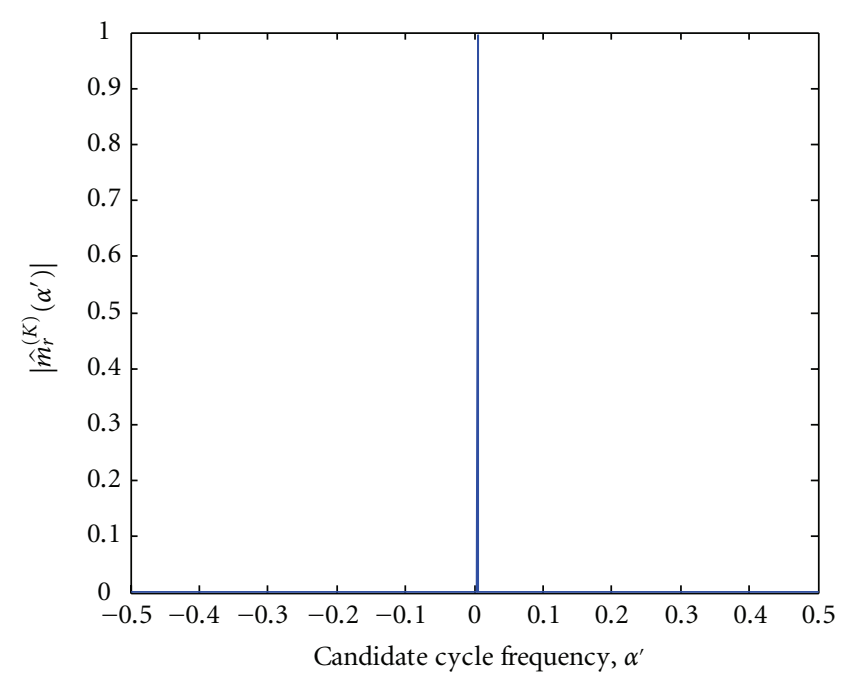

(a)

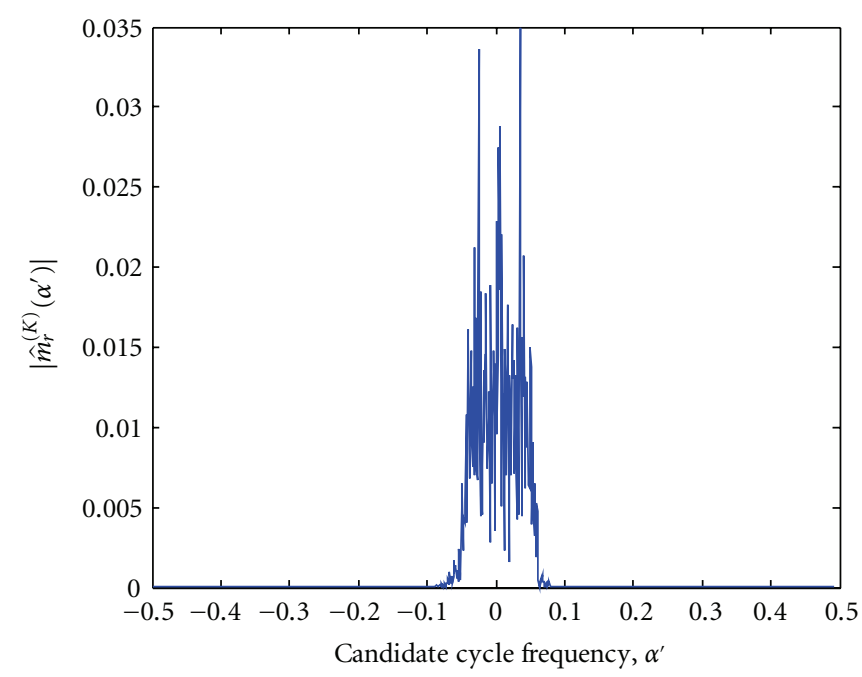

(c)

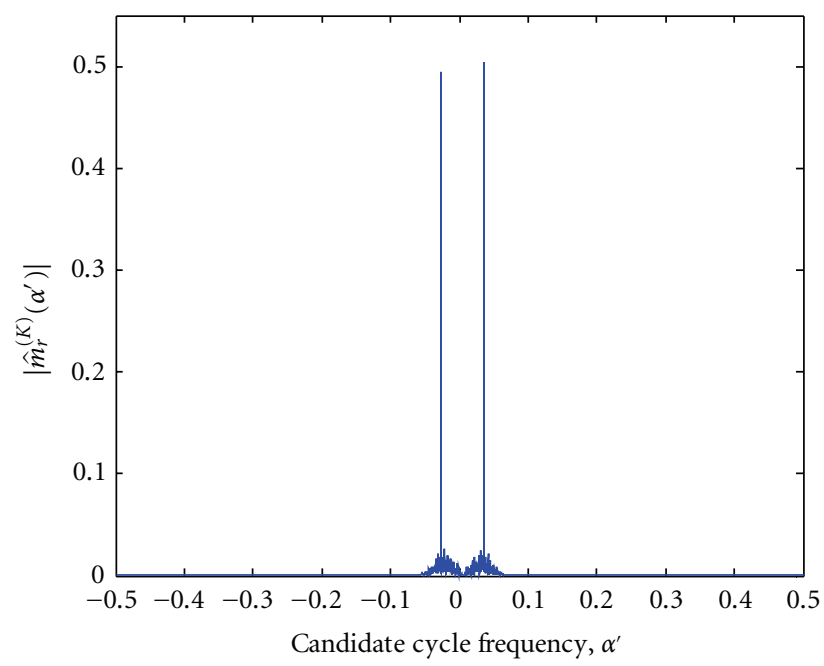

(b)

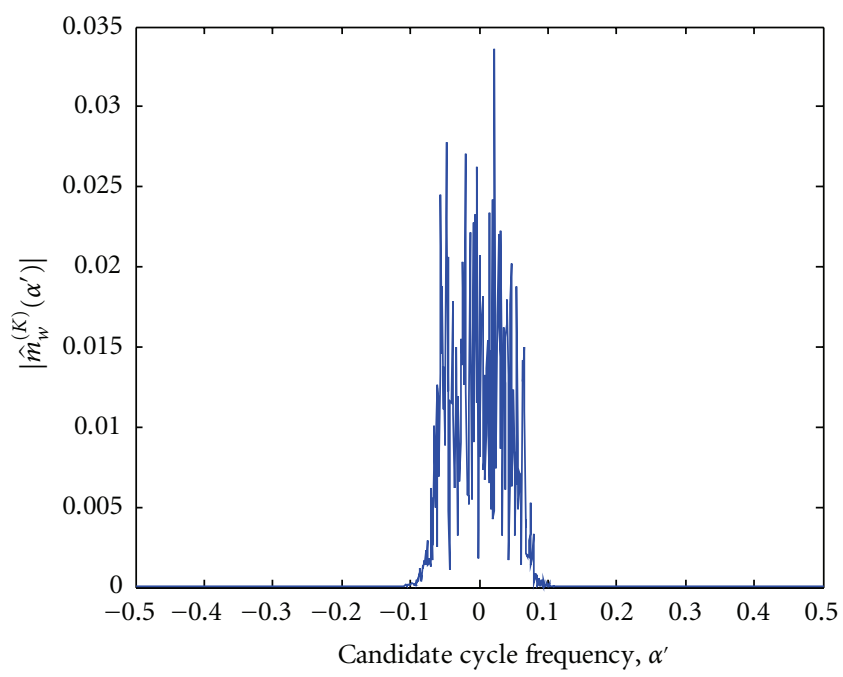

(d)

FIgure 3: The magnitudes of the first-order estimated CM at candidate CFs $\alpha^{\prime}, \alpha^{\prime} \in[-1 / 2,1 / 2$ ), for (a) AM, (b) 2-FSK, (c) 2-PSK signals at $20 \mathrm{~dB}$ SNR, and (d) noise with power $20 \mathrm{dBm}$.

CM exceed the cutoff value, applying a cyclostationarity test to find the number of first-order CFs, and using this result for decision making. Theoretically, no candidate CFs are tested for SNRs below $\mathrm{SNR}_{\text {co }}$ (with infinite length data sequence and for this SNR range, both statistically significant and insignificant $\mathrm{CM}$ values lie below $V_{\text {co }}$ and are not tested in Step 2). As a result, the probability of detection and correct classification for AM and FSK signals equals zero, that is, $P_{\mathrm{dc}}^{(\mathrm{AM} \mid \mathrm{AM})}=0$ and $P_{\mathrm{dc}}^{(M \mathrm{FSK} \mid M \mathrm{MSK})}=0$. On the other hand, theoretically, all CFs and no other candidate CFs are tested for SNRs above $\mathrm{SNR}_{\text {co }}$. Apparently, in this case, the probability of detection and correct classification for AM signals equals the probability that the corresponding CF passes the cyclostationarity test in Step 2 of the algorithm, or, in other words, the probability of detecting the
CF. Furthermore, the probability of detection and correct classification for 2-FSK and $M$-FSK $(M \geq 4)$ signals is given by the probability of detecting two and between $2^{m-1}+1$ and $2^{m} \mathrm{CFs}$, respectively. Given the assumption of independent detection of CFs, one can show that the expressions for the probabilities of detection and correct classification $P_{\mathrm{dc}}^{(\mathrm{AM} \mid \mathrm{AM})}$ and $P_{\mathrm{dc}}^{(M \mathrm{FSK} \mid M \mathrm{FSK})}$ are, respectively, given by

$$
\begin{gathered}
P_{\mathrm{dc}}^{(\mathrm{AM} \mid \mathrm{AM})}=P_{\mathrm{d}}^{(1)}, \\
P_{\mathrm{dc}}^{(M \mathrm{FSK} \mid M \mathrm{MSK})}=\prod_{\nu_{0}=1}^{M} P_{\mathrm{d}}^{\left(\nu_{0}\right)}+\sum_{m=1}^{M / 2-1} \mathcal{P}_{m},
\end{gathered}
$$




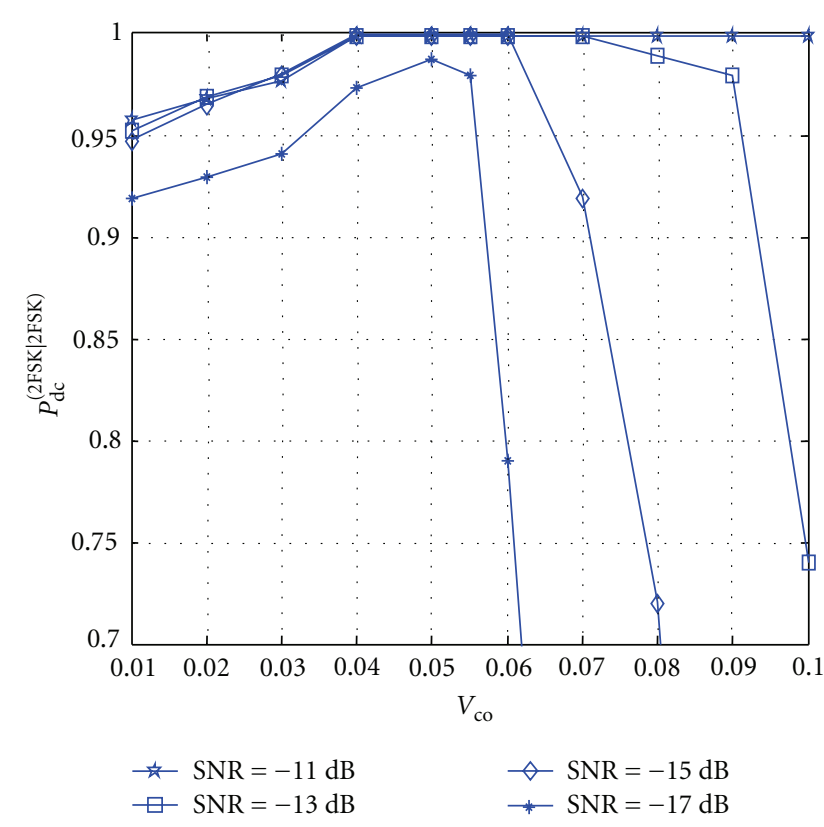

FIGURE 4: The probability of detection and correct classification, $P_{\mathrm{dc}}^{(2 \mathrm{FSK} \mid 2 \mathrm{FSK})}$, as a function of the cutoff value, $V_{\mathrm{co}}$, for several SNR values and 1-second observation interval.

where

$$
\begin{aligned}
& \mathcal{P}_{m}=\sum_{\substack{\nu_{1} \geq 1, \ldots, v_{\mathrm{m}} \geq 1 \\
\nu_{m}>\ldots>\nu_{1}}}^{M} \prod_{v_{m+1} \in \mathcal{S}_{\left(\nu_{1}, \ldots, \nu_{m}\right)}} P_{\mathrm{d}}^{\left(v_{m+1}\right)} \\
& \quad \times\left(1-P_{\mathrm{d}}^{\left(\nu_{m}\right)}\right) \cdots\left(1-P_{\mathrm{d}}^{\left(\nu_{1}\right)}\right), \\
& \delta_{\left(\nu_{1}, \ldots, v_{m}\right)}=\left\{\nu: 1 \leq \nu \leq M, \nu \neq \nu_{1}, \ldots, v_{m}\right\},
\end{aligned}
$$

and $P_{\mathrm{d}}^{(\nu)}$ is the probability of detecting the $v$ th CF (see Appendix B for details).

As the cutoff value is, theoretically, set above statistically insignificant peaks, when noise or SSB, DSB, SCLD, or CPSCLD signals are present at the receive-side, no candidate CFs are selected in Step 1 to be tested in Step 2 of the algorithm. As such, no first-order CFs are detected, and the probability of detecting noise or such signals equals one.

Note that this analysis remains valid for the benchmark, except that (17) and (18) also apply for SNRs below $\mathrm{SNR}_{\mathrm{co}}$ (no cutoff value, $V_{\text {co }}$, is employed with known CFs at the receive side).

\section{Numerical Results and Discussions}

6.1. Simulation Setup. $M$-FSK, $M=2$, 4, and 8, and AM signals with a single-sided bandwidth of $3 \mathrm{kHz}$ and unit power are simulated. Unless otherwise mentioned, the observation interval available at the receive-side is 1 second, which is equivalent to 1500 2-FSK symbols, 750 4FSK symbols, and 375 8-FSK symbols, and the frequency deviation equals $T^{-1}(l=1)$. For the AM signal, the modulating signal, $m(t)$, is obtained by lowpass filtering a sequence of zero-mean Gaussian random numbers, with unit variance. The modulation index, $\mu_{A}$, is set to 0.3 . The carrier phase, $\theta$, is uniformly distributed over $[-\pi, \pi)$, the carrier frequency offset, $\Delta f$, is equal to $240 \mathrm{~Hz}$, and the time delay, $t_{0}$, is equal to $0.6 T$ and $10 f_{s}^{-1}$ for the $M$-FSK and AM signals, respectively. The received signals are sampled at rate $f_{s}=48 \mathrm{kHz}$, and the cutoff value, $V_{\text {co }}$, is set to 0.05 , unless otherwise mentioned. The threshold $\Gamma$ used for CF detection with the cyclostationarity test in Step 2 of the algorithm is set to 15.202 , which corresponds to an (asymptotic) probability of false alarm of $5 \times 10^{-4}$ [34]. The probability of detection and correct classification, used as a performance measure, is estimated from 1000 Monte Carlo trials.

\subsection{Simulation Results}

6.2.1. Estimated First-Order CM Magnitude. In Figure 2, the magnitudes of the first-order estimated CM of 4-FSK signals, $\left|\hat{m}_{r}^{(K)}\left(\alpha^{\prime}\right)\right|$, are, respectively, plotted versus candidate CFs, $\alpha^{\prime} \in[-1 / 2,1 / 2)$, for several different SNRs. The peaks in $\left|\hat{m}_{r}^{(K)}\left(\alpha^{\prime}\right)\right|$ at $\alpha^{\prime}=\alpha$ decrease with SNR, until they become comparable with the statistically insignificant peaks, which occur at $\alpha^{\prime} \neq \alpha$. By using (12), a value of $-13.8 \mathrm{~dB}$ can be obtained for $\mathrm{SNR}_{\mathrm{co}}$. As expected from the theoretical analysis, the estimated CM magnitude at $\mathrm{CF}, \alpha$, is around 0.25 at $20 \mathrm{~dB}$ SNR (see Figure 2(a)). In addition, for SNRs well above $\mathrm{SNR}_{\mathrm{co}}$, the estimated CM magnitudes lie above $V_{\text {co }}$ (see Figures 2(a) and 2(b)) for all $M$ CFs, at SNRs around $\mathrm{SNR}_{\mathrm{co}}$, these are near $V_{\mathrm{co}}$ (see Figure 2(c)), and for SNRs well below $\mathrm{SNR}_{\mathrm{co}}$, they all drop below $V_{\text {co }}$ (see Figure 2(d)). In Figures 3(a)-3(c), the magnitudes of the first-order estimated CMs of AM, 2FSK, and 2-PSK signals, $\left|\hat{m}_{r}^{(K)}\left(\alpha^{\prime}\right)\right|$, are, respectively, plotted versus candidate CFs, $\alpha^{\prime} \in[-1 / 2,1 / 2)$, at $20 \mathrm{~dB}$ SNR. Results for noise only, $\left|\hat{m}_{w}^{(K)}\left(\alpha^{\prime}\right)\right|$, are presented in Figure $3(\mathrm{~d})$, for $20 \mathrm{dBm}$ noise power. One can notice the peaks in $\left|\hat{m}_{r}^{(K)}\left(\alpha^{\prime}\right)\right|$ of AM and 2-FSK signals at $\alpha^{\prime}=\alpha$, with magnitudes around 1 and 0.5 , respectively. On the other hand, no such peaks are seen for 2-PSK and noise, and the magnitude of the statistically insignificant peaks lie below the cutoff value.

Further comments can be made regarding the estimation of the first-order CM magnitudes: (i) this estimation is done by using (9), which practically represents the discrete Fourier transform (DFT) of the data sequence; the DFT of the signal is empirically employed to identify the modulation order of FSK signals in [10], and a connection with this work can be inferred; (ii) efficient implementations of the DFT, specifically the various forms of the fast Fourier transform, such as those described in [35], can be used to reduce computational cost. In this paper, the standard CooleyTukey radix-2 decimation in time fast Fourier transform algorithm is used to calculate $K$ point DFTs, with only the $N$ points of the resulting $K$ point frequency domain spectrum corresponding to the signal bandwidth being used. As such, the computational complexity is of $\operatorname{order} \mathcal{O}(K \log K)$. 


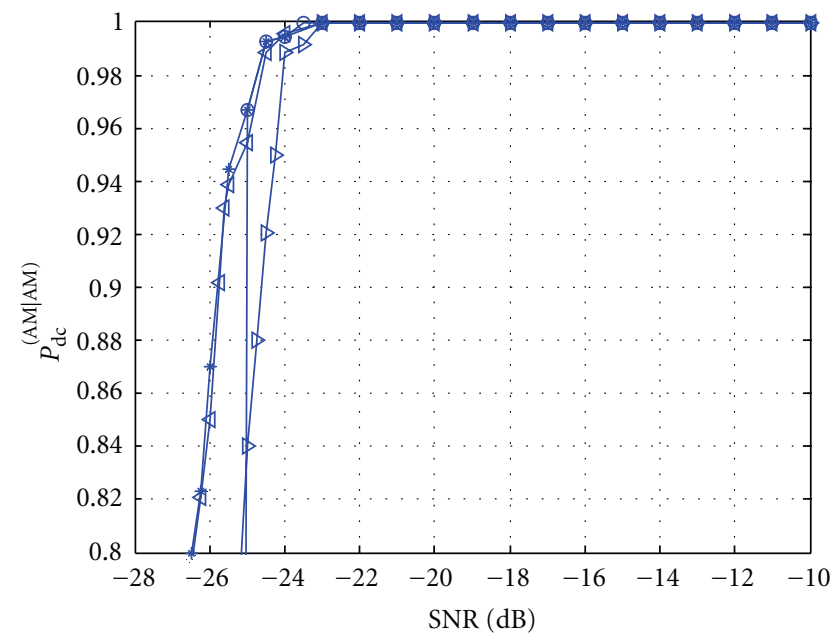

(a)

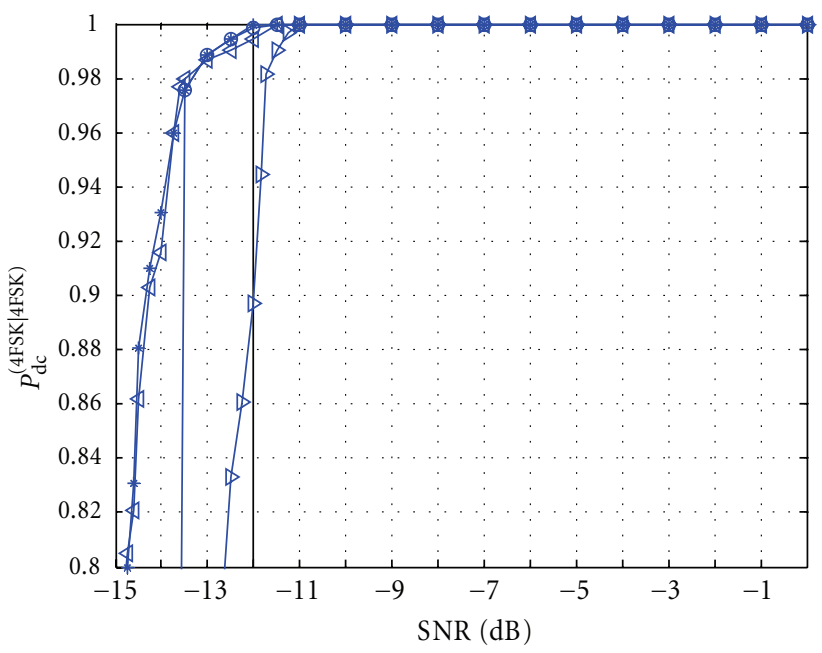

$\rightarrow$ * Known CFs, theoretical -0 Unknown CFs, theoretical

(c)

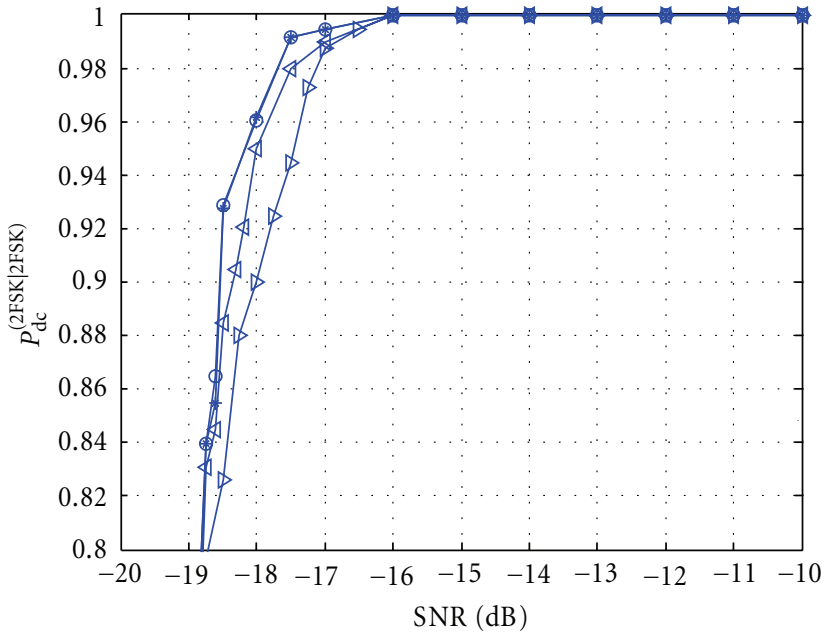

(b)

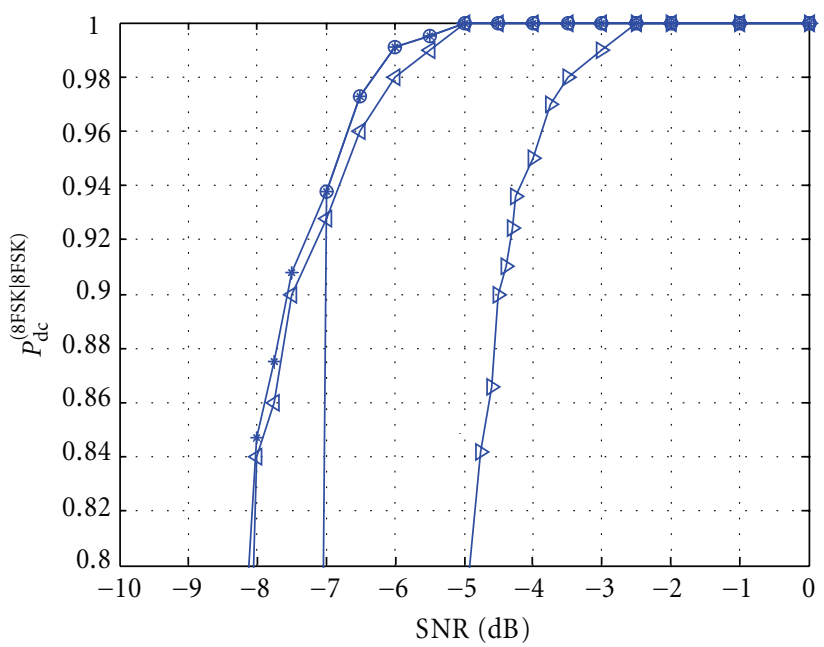

$\rightarrow$ Known CFs, theoretical -0 Unknown CFs, theoretical

(d)

FIGURE 5: The probability of detection and correct classification, (a) $P_{\mathrm{dc}}^{(\mathrm{AM} \mid \mathrm{AM})}$, (b) $P_{\mathrm{dc}}^{(2 \mathrm{FSK} \mid 2 \mathrm{FSK})}$, (c) $P_{\mathrm{dc}}^{(4 \mathrm{FSK} \mid 4 \mathrm{FSK})}$, and (d) $P_{\mathrm{dc}}^{(8 \mathrm{FSK} \mid 8 \mathrm{FSK})}$ versus SNR, with 1-second observation interval.

6.2.2. Setting the Cutoff Value. The cutoff value, $V_{\mathrm{co}}$, is empirically set based on the study of the statistically insignificant peaks for signals of interest, including $\mathrm{AM}$, $M$-FSK, $M=2,4,8$, and noise, for a diverse selection of signal parameters such as bandwidth and frequency deviation, sampling frequency, observation interval, and SNR. Examples of the cutoff values are given in Table 1 for different observation intervals (number of samples). For these results, the sampling frequency was set to 16 times the bandwidth, and frequency deviation to $l T^{-1}, l=$ $1,2,3$. Regardless of the SNR, increasing the observation interval allows a lower cutoff value, as the CM estimates are more accurate (asymptotically, the CM magnitudes corresponding to the noise floor go to zero). Figure 4 shows the performance achieved for correctly detecting and classifying 2-FSK signals, $P_{\mathrm{dc}}^{(2 \mathrm{FSK} \mid 2 \mathrm{FSK})}$, as a function of the cutoff value and for different SNR values. Note that as the SNR decreases, the performance is severely degraded by an increase in the cutoff value. This result is expected since a higher cutoff value leads to a higher $\mathrm{SNR}_{\mathrm{co}}$, and, as the SNR decreases, the statistically significant peaks are missed in Step 1 of the algorithm. On the other hand, the performance is only slightly degraded for lower cutoff values. The $\mathrm{SNR}_{\mathrm{co}}$ decreases as $V_{\text {co }}$ decreases, and the statistically significant peaks lie above the cutoff value. However, statistically insignificant peaks also exceed the 
TABLE 1: Examples of cutoff values for several observation intervals.

\begin{tabular}{lc}
\hline $\begin{array}{l}\text { Observation interval } \\
\left(\text { number of samples } \times 10^{3}\right)\end{array}$ & Cutoff value, $\mathrm{V}_{\text {co }}$ \\
\hline 36 to 48 & 0.055 \\
48 to 60 & 0.050 \\
60 to 72 & 0.045 \\
96 to 108 & 0.003 \\
\hline
\end{tabular}

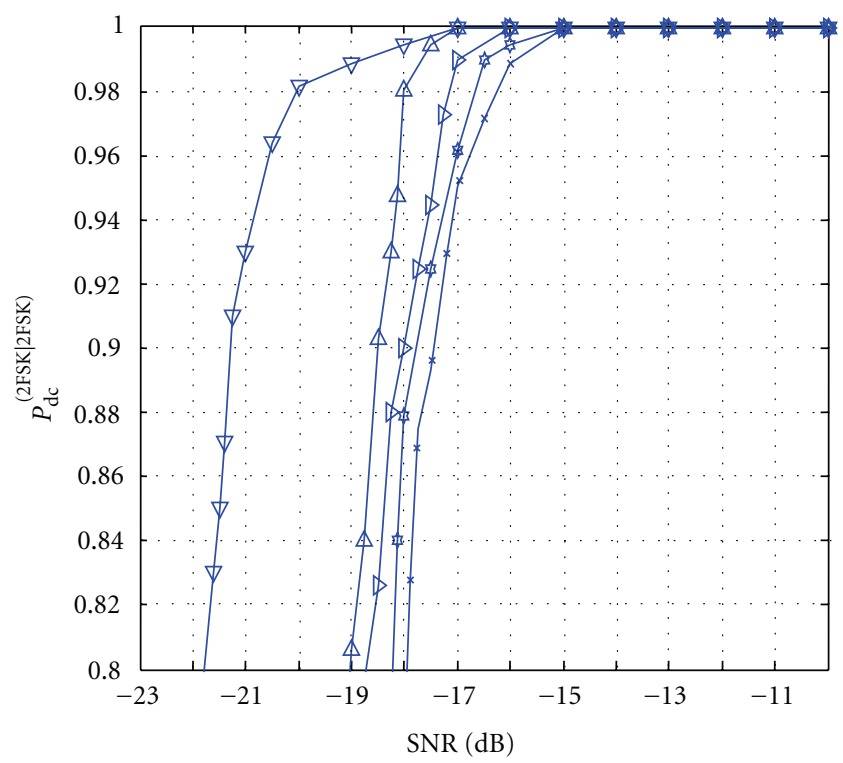

$$
\begin{aligned}
& \nabla 2 \mathrm{~s}, V_{\mathrm{co}}=0.03, f_{d}=1 / T \quad \longrightarrow 1 \mathrm{~s}, V_{\mathrm{co}}=0.05, f_{d}=2 / T \\
& \triangle 1.25 \mathrm{~s}, V_{\mathrm{co}}=0.045, f_{d}=1 / T \quad \nVdash 1 \mathrm{~s}, V_{\mathrm{co}}=0.05, f_{d}=3 / T \\
& \triangleright 1 \mathrm{~s}, V_{\mathrm{co}}=0.05, f_{d}=1 / T
\end{aligned}
$$

Figure 6: The probability of detection and correct signal classification, $P_{\mathrm{dc}}^{(2 \mathrm{FSK} \mid 2 \mathrm{FSK})}$, versus SNR, for several observation intervals and frequency deviations.

cutoff value and are selected to be tested in Step 2 of the algorithm. Reducing the cutoff value increases the number of statistically insignificant peaks selected, but most of these peaks do not pass the cyclostationarity test in Step 2, and the degradation in performance is not significant. However, the increase in the number of tested peaks does increase the computational cost. For the case under study, a cutoff value of 0.05 is a reasonable choice, as this provides a low $\mathrm{SNR}_{\mathrm{co}}$ and minimizes the selection of statistically insignificant peaks in Step 1 of the algorithm.

6.2.3. Performance of the Proposed Algorithm and Comparison Against the Benchmark. Performance results obtained from both theoretical performance analysis and simulations of the proposed algorithm and benchmark are presented in Figures 5 and 6 , where $P_{\mathrm{dc}}^{(\mathrm{AM} \mid \mathrm{AM})}$ and $P_{\mathrm{dc}}^{(M \mathrm{FSK} \mid M \mathrm{FSK})}$ are plotted as a function of SNR. Several conclusions can be inferred: (i) for the same observation interval, a specified performance can be achieved with a lower SNR for AM and lower-order FSK modulated signals; (ii) the performance differential for the proposed algorithm and benchmark increases with the FSK signal modulation order. This behavior is attributed to the decreased accuracy of the CM estimation resulting from the decrease in the number of symbols for a given observation interval available at the receive-side (see Section 6.1 for the simulation setup). This leads to more CFs being missed in Step 1 as the SNR decreases, and consequently, to a degradation of the classification performance in the absence of a priori knowledge of the CFs; (iii) the simulation results are very close to the theoretical predictions for the benchmark over the entire SNR range, whereas this is valid for the proposed algorithm at SNRs well above $\mathrm{SNR}_{\mathrm{co}}$. The latter behavior is an expected consequence of assuming that all statistically significant peaks exceed $V_{\text {co }}$ for SNRs above $\mathrm{SNR}_{\mathrm{co}}$ in the theoretical performance analysis. As shown in Figure 2(c), for $\mathrm{SNR}$ close to $\mathrm{SNR}_{\mathrm{co}}$, statistically significantly peaks can be missed in Step 1. Figure 6 presents simulation results for 2-FSK signal detection and classification for different observation intervals, obtained by varying the number of symbols. As expected, improved performance is obtained with a longer observation interval, since a lower cutoff value can be set, thus allowing a reduction in $\mathrm{SNR}_{\mathrm{co}}$. In addition, results for different frequency deviations are shown for a given observation interval. Interestingly, the results obtained for larger frequency deviations $(l=$ $2,3)$ are relatively close to those obtained for $l=1$. In addition, we have simulated scenarios when only noise or other signals, such as 4-PSK, 16-QAM, 64-QAM, SSB, and DSB, are present, and have estimated the average probability for deciding that no FSK and AM signal is present. For SNRs above $-20 \mathrm{~dB}$, this probability is close to one.

\section{Conclusions}

An algorithm based on first-order cyclostationarity has been developed for the joint detection and classification of FSK and AM signals. Theoretical analysis and simulation experiments demonstrate that the algorithm is able to discriminate between AM and FSK modulation types with minimal requirements for a priori information about the signal parameters. A comparison of these results with a performance benchmark, based on the assumption of additional a priori signal parameter information being available at the receive-side, demonstrates that the algorithm performs reasonably well. Future work will address additional issues of interest, such as a theoretical analysis of the minimum length of the observation interval required at the receiveside to attain a specified performance at a given SNR, the investigation and comparison of diverse methods for detecting the existence/number of cycle frequencies in the received signal, the extension to other modulation types, and more complex propagation environments. 


\section{Appendices}

\section{A. First-Order Cyclostationarity of M-FSK and AM Signals Affected by Gaussian Noise, Phase, Frequency Offset, and Time Delay}

The first-order time-varying moment of the $M$-FSK signals is expressed as

$$
\begin{aligned}
\tilde{m}_{r}(t) & =\mathrm{E}[r(t)] \\
= & \sqrt{8} e^{j \theta} M^{-1} \\
& \times \sum_{m=1}^{M} \sum_{i} e^{j 2 \pi f_{\Delta} \tilde{s}_{m}(t-i T-\varepsilon T)} g\left(t-i T-t_{0}\right) e^{j 2 \pi \Delta f t},
\end{aligned}
$$

where it is assumed that $\mathcal{A}=\sqrt{8}$. The average is performed with respect to the unknown data symbols, under the assumption that the symbol over the $i$ th period takes equiprobable values in the signal alphabet, $A_{M-F S K}$.

Equation (A.1) can be further written as

$$
\tilde{m}_{r}(t)=C \sum_{m=1}^{M}\left(e^{j 2 \pi f_{\Delta} \tilde{s}_{m} t} g(t) \bigotimes \sum_{i} \delta\left(t-i T-t_{0}\right)\right) e^{j 2 \pi \Delta f t},
$$

where $\mathcal{C}=\sqrt{\delta} e^{j \theta} M^{-1}, \delta(t)$ is the Dirac delta function, and $\otimes$ is the convolution operator.

If $\Delta f=0, \tilde{m}_{r}(t)$ is seen to be a periodic function of $t$, with fundamental period $T$ [20]. In this case, the first-order time-varying moment can be easily expressed as a Fourier series. Such an expression is derived in [20], for $t_{0}=0$ and $g(t)=u_{T}(t)$. On the other hand, with $\Delta f \neq 0$, the Fourier transform of (A.2) yields

$$
\begin{aligned}
\Im & \left\{\tilde{m}_{r}(t)\right\} \\
= & \mathcal{C} \int_{-\infty}^{\infty} \sum_{m=1}^{M}\left(e^{j 2 \pi f_{\Delta} \tilde{s}_{m} t} g(t) \otimes \sum_{i} \delta\left(t-i T-t_{0}\right)\right) \\
& \times e^{j 2 \pi \Delta f t} e^{-j 2 \pi \tilde{\alpha} t} d t \\
= & \mathcal{C} \iint_{-\infty}^{\infty} \sum_{m=1}^{M} e^{j 2 \pi f_{\Delta} \tilde{s}_{m} v} g(v) \\
& \times \sum_{i} \delta\left(t-v-i T-t_{0}\right) e^{-j 2 \pi(\tilde{\alpha}-\Delta f) t} d v d t \\
= & C \int_{-\infty}^{\infty} \sum_{m=1}^{M} e^{j 2 \pi f_{\Delta} \tilde{s}_{m} v} g(v) \\
& \times \int_{-\infty}^{\infty} \sum_{i} \delta\left(t-v-i T-t_{0}\right) e^{-j 2 \pi(\tilde{\alpha}-\Delta f) t} d t d v
\end{aligned}
$$

$$
\begin{aligned}
= & \mathcal{C} \int_{-\infty}^{\infty} \sum_{m=1}^{M} e^{j 2 \pi f_{\Delta} \tilde{s}_{m} v} g(v) \\
& \times \int_{-\infty}^{\infty} \sum_{i} \delta(u-i T) e^{-j 2 \pi(\tilde{\alpha}-\Delta f) u} d u e^{-j 2 \pi(\tilde{\alpha}-\Delta f)\left(v+t_{0}\right)} d v \\
= & \mathcal{C} \int_{-\infty}^{\infty} \sum_{m=1}^{M} e^{j 2 \pi f_{\Delta} \tilde{s}_{m} v} e^{-j 2 \pi(\tilde{\alpha}-\Delta f) v} g(v) d v \\
& \times T^{-1} \sum_{i} \delta\left(\widetilde{\alpha}-\Delta f-i T^{-1}\right) e^{-j 2 \pi(\tilde{\alpha}-\Delta f) t_{0},}
\end{aligned}
$$

where $\mathfrak{I}\{\cdot\}$ denotes the Fourier transform. Convolution and change of variables are, respectively, performed at the second and fourth steps in the right hand-side of (A.3), and the identity $\mathfrak{I}\left\{\sum_{i} \delta(u-i T)\right\}=T^{-1} \sum_{i} \delta\left(\tilde{\alpha}-i T^{-1}\right)$ is used in the fifth step. Note that $\mathfrak{I}\left\{\tilde{m}_{r}(t)\right\} \neq 0$ if

$$
\tilde{\alpha}=\Delta f+i T^{-1}, \quad i \text { integer. }
$$

The $\tilde{\alpha}$-frequency domain is thus seen to be discrete. By substituting (A.4) into (A.3), we obtain

$$
\begin{aligned}
\mathfrak{I}\left\{\tilde{m}_{r}(t)\right\} & \\
= & \mathcal{C} \sum_{m=1}^{\mathrm{M}} \sum_{i} \delta\left(\tilde{\alpha}-\Delta f-i T^{-1}\right) e^{-j 2 \pi i t_{0} T^{-1}} T^{-1} \\
& \times \int_{-\infty}^{\infty} g(v) e^{-j 2 \pi\left(f_{\Delta} \tilde{s}_{m}-i T^{-1}\right) v} d v .
\end{aligned}
$$

If the product $f_{\Delta} \widetilde{s}_{m}$ is an integer of $T^{-1}$, that is, $f_{\Delta} \widetilde{s}_{m}=p T^{-1}$, with $p$ as an integer, then the integral on the right side of (A.5) equals $g\left((p-i) T^{-1}\right)$, with $g(f)$ as the Fourier transform of $g(t)$. Since the receive filter passes the signal without attenuation over the effective frequency range, the nulls of $g(f)$ will be nearly the same as the frequency nulls of $u_{T}(t)$. Thus, $g\left((p-i) T^{-1}\right)$ equals $T$ if $p=i$ and 0 otherwise, and (A.5) becomes

$$
\mathfrak{I}\left\{\tilde{m}_{r}(t)\right\}=\mathcal{C} \sum_{p \in P} e^{-j 2 \pi p t_{0} T^{-1}} \delta\left(\tilde{\alpha}-\Delta f-p T^{-1}\right),
$$

where $P=\left\{p: p\right.$ integer, $\left.p=f_{\Delta} \widetilde{s}_{m} T, \widetilde{s}_{m} \in A_{M-\mathrm{FSK}}\right\}$.

If $f_{\Delta}=l T^{-1}$, with $l$ as an integer, it follows that $p=$ $\pm l, \pm 3 l, \ldots, \pm(M-1) l$, and the spectrum consists of $M$ finitestrength additive components,

$$
\Im\left\{\tilde{m}_{r}(t)\right\}=\sum_{p \in\{ \pm l, \pm 3 l, \ldots, \pm(M-1) l\}} \mathcal{C} e^{-j 2 \pi p t_{0} T^{-1}} \delta\left(\tilde{\alpha}-\Delta f-p T^{-1}\right) .
$$

The expression for $\tilde{m}_{r}(t)$ thus becomes

$$
\tilde{m}_{r}(t)=\sum_{p \in\{ \pm l, \pm 3 l, \ldots, \pm(M-1) l\}} \mathcal{C} e^{-j 2 \pi p t_{0} T^{-1}} e^{j 2 \pi\left(\Delta f+p T^{-1}\right) t} .
$$

This is seen to be equivalent to (5), with $\tilde{\kappa}=\{\Delta f+$ $\left.\tilde{\gamma}, \tilde{\gamma}=p T^{-1}, p= \pm l, \pm 3 l, \ldots, \pm(M-1) l\right\}$, and 
$\tilde{m}_{r}(\tilde{\alpha})=\sqrt{8} M^{-1} e^{j \theta} e^{-j 2 \pi p t_{0} T^{-1}}$. Note that the same expression for the first-order $\mathrm{CM}$ at $\mathrm{CF} \tilde{\alpha}, \tilde{m}_{r}(\widetilde{\alpha})$, can be obtained by applying (6). From the results previously derived for the continuous-time signal, $r(t),(10)-(11)$ can be obtained for the normalized discrete-time signal, $r[k]=$ $\left.r(t)\right|_{t=k f_{s}^{-1}} / \sqrt{8+\mathcal{N}}$, by applying (7) and (8), and taking into account the signal normalization.

With the signal model given in (3), a similar analysis can be easily performed for AM signal, leading to results presented in (13) and (14).

\section{B. Cyclostationarity Test and Probability of CF Detection}

A.1. Cyclostationarity Test. With this cyclostationarity test, the presence of a CF is formulated as a binary hypothesistesting problem; that is, under hypothesis $H_{0}$ the tested candidate $\mathrm{CF}, \alpha^{\prime}$, is not a $\mathrm{CF}, \alpha$, and under hypothesis $H_{1}$ the tested candidate $\mathrm{CF}, \alpha^{\prime}$, is a CF, $\alpha$. This test is applied here to first-order candidate CFs (In [33], the test is developed for real-valued signals. However, it can easily be shown that this remains valid for complex-valued signals.) and consists of the following three steps [33].

(i) The first-order $\mathrm{CM}$ at candidate $\mathrm{CF}, \alpha^{\prime}$, is estimated from $K$ samples. The vector

$$
\hat{\mathbf{m}}_{r}^{(K)}=\left[\operatorname{Re}\left\{\hat{m}_{r}^{(K)}\left(\alpha^{\prime}\right)\right\} \operatorname{Im}\left\{\hat{m}_{r}^{(K)}\left(\alpha^{\prime}\right)\right\}\right]
$$

is formed, with $\operatorname{Re}\{\cdot\}$ and $\operatorname{Im}\{\cdot\}$ as the real and imaginary parts, respectively.

(ii)The statistic

$$
\mathcal{T}^{(K)}=K \hat{\mathbf{m}}_{r}^{(K)} \hat{\Sigma}_{r}^{-1} \hat{\mathbf{m}}_{r}^{(K) \dagger}
$$

is computed for the candidate $\mathrm{CF}, \alpha^{\prime}$. Here the superscripts -1 and $\dagger$ denote matrix inverse and transpose, respectively, and $\hat{\Sigma}_{r}$ is an estimate of the covariance matrix

$$
\Sigma_{r}=\left[\begin{array}{ll}
\operatorname{Re}\left\{\frac{\mathcal{Q}_{2,0}+\mathcal{Q}_{2,1}}{2}\right\} & \operatorname{Im}\left\{\frac{\mathcal{Q}_{2,0}-\mathcal{Q}_{2,1}}{2}\right\} \\
\operatorname{Im}\left\{\frac{\mathcal{Q}_{2,0}+\mathcal{Q}_{2,1}}{2}\right\} & \operatorname{Re}\left\{\frac{\mathcal{Q}_{2,1}-\mathcal{Q}_{2,0}}{2}\right\}
\end{array}\right],
$$

where covariances $\mathcal{Q}_{2,0}$ and $\mathcal{Q}_{2,1}$ are, respectively, given as $\lim _{K \rightarrow \infty} K \operatorname{Cum}\left[\hat{m}_{r}^{(K)}\left(\alpha^{\prime}\right), \hat{m}_{r}^{(K)}\left(\alpha^{\prime}\right)\right]$ and $\lim _{K \rightarrow \infty} K \operatorname{Cum}\left[\hat{m}_{r}^{(K)}\left(\alpha^{\prime}\right), \hat{m}_{r}^{(K)^{*}}\left(\alpha^{\prime}\right)\right]$, with ${ }^{*}$ as complex conjugate and $\operatorname{Cum}[\cdot]$ as the cumulant operator.

For the estimators of these covariances, see, for example, (48) in [33].

The statistic $\mathcal{T}^{(K)}$ has an (asymptotic) chi-square distribution with two degrees of freedom under the hypothesis $H_{0}$, whereas an (asymptotic) normal distribution under $H_{1}$ [33].

(iii)The decision on the candidate CF, $\alpha^{\prime}$, is made by comparing the statistic $\mathcal{T}^{(K)}$ against a threshold, $\Gamma$. This threshold is set for a specific (asymptotic) probability of false alarm, defined as $\operatorname{Pr}\left\{\mathcal{T}^{(K)} \geq \Gamma \mid H_{0}\right\}$, under the assumption that the number of samples goes to infinity, and found from the tables of the chi-squared distribution. If $\mathcal{T}^{(K)} \geq \Gamma$, the tested candidate is decided to be a CF, otherwise not.
B.2. Probability of CF Detection. With the previously presented cyclostationarity test, the probability of deciding that a CF is indeed a CF, which is referred to as the probability of $\mathrm{CF}$ detection, is given as

$$
P_{\mathrm{d}}=\operatorname{Pr}\left\{\mathcal{T}^{(K)} \geq \Gamma \mid H_{1}\right\} .
$$

Based on the fact that the (asymptotic) distribution of $\mathcal{T}^{(K)}$ under the hypothesis $H_{1}$ is normal, with mean $m_{\mathcal{T}}=$ $K \mathbf{m}_{r} \Sigma_{r}^{-1} \mathbf{m}_{r}^{\dagger}$ and variance $\sigma_{\mathcal{T}}^{2}=4 K \mathbf{m}_{r} \Sigma_{r}^{-1} \mathbf{m}_{r}^{\dagger}$ [33], the expression for the (asymptotic) probability of CF detection is given by

$$
P_{\mathrm{d}}=\int_{\left(\Gamma-m_{\mathcal{T}}\right) / \sigma_{\mathcal{T}}}^{\infty}(2 \pi)^{-1 / 2} e^{-y^{2} / 2} d y
$$

\section{List of Abbreviations}

$\begin{array}{ll}\text { AM: } & \text { Amplitude modulation } \\ \text { CF: } & \text { Cycle frequency } \\ \text { CM: } & \text { Cyclic moment } \\ \text { CP-SCLD: } & \text { Cyclically prefixed single carrier linear digital } \\ \text { DFT: } & \text { Discrete Fourier transform } \\ \text { DSB: } & \text { Double side-band } \\ \text { FB: } & \text { Feature-based } \\ \text { FSK: } & \text { Frequency shift keying } \\ \text { LB: } & \text { Likelihood-based } \\ \text { PSK: } & \text { Phase shift keying } \\ \text { QAM: } & \text { Quadrature amplitude modulation } \\ \text { SCLD: } & \text { Single carrier linear digital } \\ \text { SNR: } & \text { Signal-to-noise ratio } \\ \text { SSB: } & \text { Single side-band } \\ \text { UHF: } & \text { Ultra high frequency } \\ \text { VHF: } & \text { Very high frequency. }\end{array}$

\section{Acknowledgment}

This work has been supported by Defence Research and Development Canada.

\section{References}

[1] J. Mitola III, "Cognitive radio for flexible mobile multimedia communications," in Proceedings of the IEEE International Workshop on Mobile and Multimedia Communications, pp. 310, San Diego, Calif, USA, 1999.

[2] S. Haykin, "Cognitive radio: brain-empowered wireless communications," IEEE Journal on Selected Areas in Communications, vol. 23, pp. 201-220, 2005.

[3] Federal Communication Commission, "Spectrum policy task force," ET Docket No. 02-155, November 2002.

[4] O. A. Dobre, A. Abdi, Y. Bar-Ness, and W. Su, "Survey of automatic modulation classification techniques: classical approaches and new trends," IET Communications, vol. 1, pp. 137-156, 2007.

[5] B. F. Beidas and C. L. Weber, "Higher-order correlation-based approach to modulation classification of digitally frequencymodulated signals," IEEE Journal on Selected Areas in Communications, vol. 13, pp. 89-101, 1995.

[6] B. F. Beidas and C. L. Weber, "Asynchronous classification of MFSK signals using the higher order correlation domain," 
IEEE Transactions on Communications, vol. 46, no. 4, pp. 480493, 1998.

[7] S. Z. Hsue and S. S. Soliman, "Automatic modulation classification using zero crossing," IEE Proceedings F, vol. 137, no. 6, pp. 459-464, 1990.

[8] K. C. Ho, W. Prokopiw, and Y. T. Chan, "Modulation identification of digital signals by the wavelet transform," IEE Proceedings: Radar, Sonar and Navigation, vol. 147, pp. 169 176, 2000.

[9] A. V. Rosti and V. Koivunen, "Classification of MFSK modulated signals using the mean of complex envelope," in Proceedings of the European Signal Processing Conference (EUSIPCO '00), pp. 581-584, 2000.

[10] Z. Yu, Y. Q. Shi, and W. Su, "M-ary frequency shift keying signal classification based-on discrete Fourier transform," in Proceedings of the IEEE Military Communications Conference (MILCOM '03), vol. 2, pp. 1167-1172, 2003.

[11] E. E. Azzouz and A. K. Nandi, Automatic Modulation Recognition of Communication Signals, Kluwer Academic Publishers, Dordrecht, The Netherlands, 1996.

[12] W. A. Gardner, "Signal interception: a unifying theoretical framework for feature detection," IEEE Transactions on Communications, vol. 36, pp. 897-906, 1988.

[13] C. M. Spooner, "Classification of cochannel communication signal using cyclic cumulants," in Proceedings of the Asilomar Conference on Signals, Systems and Computers (ASILOMAR '95), pp. 531-536, 1995.

[14] C. M. Spooner, W. A. Brown, and G. K. Yeung, "Automatic radio-frequency environment analysis," in Proceedings of the Asilomar Conference on Signals, Systems and Computers (ASILOMAR '00), vol. 2, pp. 1181-1186, 2000.

[15] O. A. Dobre, Y. Bar-Ness, and W. Su, "Robust QAM modulation classification algorithm using cyclic cumulants," in Proceedings of the IEEE Wireless Communications and Networking Conference (WCNC'04), vol. 2, pp. 745-748, 2004.

[16] O. A. Dobre, A. Abdi, Y. Bar-Ness, and W. Su, "Cyclostationarity-based blind classification of analog and digital modulations," in Proceedings of the IEEE Military Communications Conference (MILCOM '06), pp. 1-7, 2006.

[17] A. Punchihewa, O. A. Dobre, S. Rajan, and R. Inkol, "Cyclostationarity-based algorithm for blind recognition of OFDM and single carrier linear digital modulations," in Proceedings of the IEEE International Symposium on Personal, Indoor and Mobile Radio Communications (PIMRC '07), pp. $1-5,2007$.

[18] O. A. Dobre, Q. Zhang, S. Rajan, and R. Inkol, "Second-order cyclostationarity of cyclically prefixed single carrier linear digital modulations with applications to signal recognition," in Proceedings of the IEEE Global Telecommunications Conference (GLOBECOM '08), pp. 3513-3517, 2008.

[19] F. Gini and G. B. Giannakis, "Frequency offset and symbol timing recovery in flat-fading channels: a cyclostationary approach," IEEE Transactions on Communications, vol. 46, pp. 400-411, 1998.

[20] A. Ferréol, P. Chevalier, and L. Albera, "Second-order blind separation of first- and second-order cyclostationary sourcesapplication to AM, FSK, CPFSK, and deterministic sources," IEEE Transactions on Signal Processing, vol. 52, pp. 845-861, 2004.

[21] M. Shi, Y. Bar-Ness, and W. Su, "Blind OFDM systems parameters estimation for software defined radio," in Proceedings of the 2nd IEEE International Symposium on New Frontiers in Dynamic Spectrum Access Networks (DySpan '07), pp. 119-122, 2007.
[22] H. Li, Y. Bar-Ness, A. Abdi, O. S. Somekh, and W. Su, "OFDM modulation classification and parameters extraction," in Proceedings of the 1st International Conference on Cognitive Radio Oriented Wireless Networks and Communications (CROWNCOM '06), pp. 1-6, 2006.

[23] K. Kim, C. M. Spooner, I. Akbar, and J. H. Reed, "Specific emitter identification for cognitive radio with application to IEEE 802.11," in Proceedings of the IEEE Global Telecommunications Conference (GLOBECOM '08), pp. 2099-2103, 2008.

[24] K. Kim, I. A. Akbar, K. K. Bae, J. S. Um, C. M. Spooner, and J. H. Reed, "Cyclostationary approaches to signal detection and classification in cognitive radio," in Proceedings of the 2nd IEEE International Symposium on New Frontiers in Dynamic Spectrum Access Networks (DySpan '07), pp. 212-215, 2007.

[25] M. Oner and F. Jondral, "On the extraction of the channel allocation information in spectrum pooling systems," IEEE Journal on Selected Areas in Communications, vol. 25, no. 3, pp. 558-565, 2007.

[26] A. Tkachenko, D. Cabric, and R. W. Brodersen, "Cyclostationary feature detector experiments using reconfigurable BEE2," in Proceedings of the 2nd IEEE International Symposium on New Frontiers in Dynamic Spectrum Access Networks (DySpan '07), pp. 216-219, 2007.

[27] O. A. Dobre, S. Rajan, and R. Inkol, "A novel algorithm for blind recognition of $\mathrm{M}$-ary frequency shift keying modulation," in Proceedings of the IEEE Wireless Communications and Networking Conference (WCNC '07), pp. 521-525, 2007.

[28] O. A. Dobre, S. Rajan, and R. Inkol, "Exploitation of firstorder cyclostationarity for joint signal detection and classification in cognitive radio," in Proceedings of the IEEE Vehicular Technology Conference (VTC '08), pp. 1-5, 2008.

[29] A. B. Carlson, P. B. Crilly, and J. C. Rutledge, Communication Systems, McGraw Hill, New York, NY, USA, 4th edition, 2002.

[30] W. A. Gardner, Cyclostationarity in Communications and Signal Processing, IEEE Press, New York, NY, USA, 1994.

[31] A. Napolitano, "Cyclic higher-order statistics: input/output relations for discrete- and continuous-time MIMO linear almost-periodically time-variant systems," Signal Processing, vol. 42, pp. 147-166, 1995.

[32] A. V. Dandawate and G. B. Giannakis, "Asymptotic theory of mixed time averages and kth-order cyclic-moment and cumulant statistics," IEEE Transactions on Information Theory, vol. 41, pp. 216-232, 1995.

[33] A. V. Dandawate and G. B. Giannakis, "Statistical tests for presence of cyclostationarity," IEEE Transactions on Signal Processing, vol. 42, pp. 2355-2369, 1994.

[34] M. Abramowitz and I. A. Stegun, Handbook of Mathematical Functions, Dover Publications, New York, NY, USA, 1972.

[35] C. Van Loan, Computational Framework for the Fast Fourier Transform, SIAM, Philadelphia, PA, USA, 1992. 